\title{
Membrane-Type Acoustic Metamaterial with Negative Dynamic Mass
}

\author{
Z. Yang, ${ }^{*}$ Jun Mei, Min Yang, N. H. Chan, and Ping Sheng ${ }^{\dagger}$ \\ Department of Physics, Hong Kong University of Science and Technology, Clear Water Bay, Kowloon, Hong Kong, China
} (Received 1 June 2008; revised manuscript received 7 October 2008; published 14 November 2008)

\begin{abstract}
We present the experimental realization and theoretical understanding of a membrane-type acoustic metamaterial with very simple construct, capable of breaking the mass density law of sound attenuation in the $100-1000 \mathrm{~Hz}$ regime by a significant margin ( 200 times). Owing to the membrane's weak elastic moduli, there can be low-frequency oscillation patterns even in a small elastic film with fixed boundaries defined by a rigid grid. The vibrational eigenfrequencies can be tuned by placing a small mass at the center of the membrane sample. Near-total reflection is achieved at a frequency between two eigenmodes where the in-plane average of normal displacement is zero. By using finite element simulations, negative dynamic mass is explicitly demonstrated at frequencies around the total reflection frequency. Excellent agreement between theory and experiment is obtained.
\end{abstract}

DOI: 10.1103/PhysRevLett.101.204301

PACS numbers: 43.40.+s, 46.40.-f, 68.60.Bs

Acoustic or elastic metamaterials [1-5] extend the realm of elastic wave characteristics achievable by phononic crystals and natural materials. In particular, negative dynamic mass density [5] composites have demonstrated significantly subwavelength attenuation of sound in the audible regime by breaking the mass density law [1]. Here negative dynamic mass means that the spatially averaged force and acceleration are opposite in phase, and composites with such microscopic components can display macroscopic dynamics that deviates from Newton's second law [6]. In the ultimate limit of such materials, it would be desirable to have a thin and lightweight membrane with metamaterial characteristics that can operate effectively in the $100-1000 \mathrm{~Hz}$ range, the most difficult regime as dictated by the mass density law. However, stopping lowfrequency sound with a thin membrane is against simple intuition, as it is hard to imagine the elastic membrane can either effectively absorb or reflect the sound. An especially powerful argument against the latter is that total reflection requires the formation of a node at the reflecting surface; hence, a membrane with weak elastic restoring force normal to its surface is an unlikely candidate to be a lowfrequency sound reflector.

In this Letter, we present the experimental realization and theoretical understanding of a membrane-type metamaterial with negative dynamic mass characteristics, operative in the 100-1000 Hz frequency regime. By stacking such membrane-type panels with different operative frequencies, we could realize relatively broadband effectiveness. It is shown that precisely because of the weak elastic moduli of the membrane, there can be various lowfrequency oscillation patterns even within a small and finite sample with fixed boundaries as defined by a rigid grid. Such vibrational eigenmodes can be tuned by placing a small mass at the center of the membrane sample, and near-total reflection is achieved at a frequency between two eigenmodes where the in-plane average of displacement (normal to the membrane) is zero, leading to very small far-field transmission. By using finite-element simulations, negative dynamic mass is explicitly demonstrated at frequencies around the total reflection frequency. Excellent agreement between theory and experiment is shown.

The basic unit of our metamaterial consists of a circular elastic membrane (20 $\mathrm{mm}$ in diameter and $0.28 \mathrm{~mm}$ thick) with fixed boundary, imposed by a relatively rigid grid, with a small weight attached to the center. Acoustic waves are incident perpendicular to the membrane plane. Other grid shapes, such as square grids, can be used to the same effect. The central mass is a hard disk $6.0 \mathrm{~mm}$ in diameter on which Blu Tack putty can be added to increase the total mass up to $300 \mathrm{mg}$.

Measurements of the transmission amplitude and phase were conducted in a modified impedance tube apparatus, details of which can be found in [7]. Briefly stated, the apparatus consists of two Brüel and Kjær type-4026 impedance tubes with the sample sandwiched in between. The front tube has a loud speaker at one end to generate a plane wave in the tube. There are two sensors in the front tube to sense the incident and reflected waves. The third sensor in the back tube, terminated with an anechoic sponge, senses the transmitted wave. The signals from the three sensors are sufficient to resolve the transmitted and reflected wave amplitudes, in conjunction with their phases.

Figure 1(a) shows the measured transmission amplitude (solid red curve) and phase (dotted green curve) spectra. The blue dashed line indicates the mass density law [8] that is pertinent to our sample density of $0.1 \mathrm{~kg} / \mathrm{m}^{2}$. There are two peaks at 145 and $984 \mathrm{~Hz}$. But perhaps the most surprising is the dip at $237 \mathrm{~Hz}$ that breaks the mass density law by a factor of 200, implying near-total reflection by such a flimsy membrane. Below we show this phenomenon 

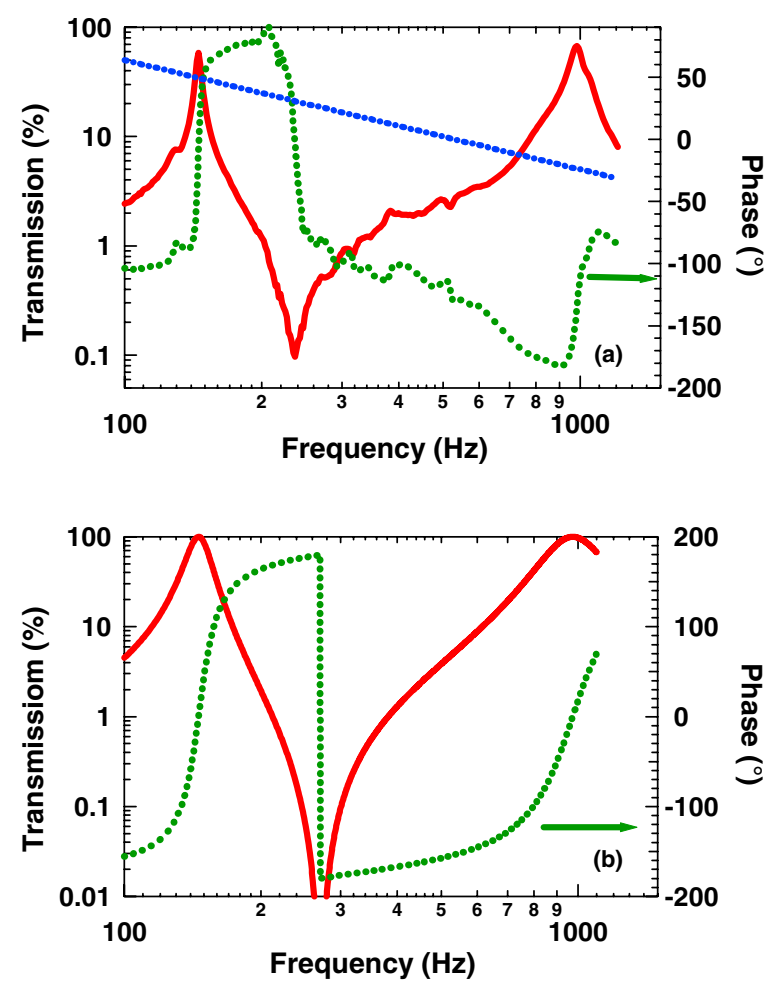

FIG. 1 (color online). (a) Experimental transmission amplitude (solid red curve) and phase (dotted green curve) of the membrane resonator. The blue dashed line indicates the transmission amplitude predicted by the mass density law with the same average area mass density as the resonator. (b) Theoretical transmission amplitude (solid red curve) and phase (dotted green curve) of the membrane resonator.

arises directly from the negative dynamic mass at this frequency, and it is an inevitable consequence of multiple low-frequency vibrational eigenmodes of the system.

In Fig. 1(b) we show the calculated transmittance amplitude (solid red curve) and phase (dotted green curve) of a circular thin rubber membrane by using COMSOL MULTIPHYSICS, a finite-element analysis and solver software package. The edge of the circular membrane was fixed, with a $6.0 \mathrm{~mm}$ diameter circular steel disk of $300 \mathrm{mg}$ fixed at the center. In our calculation, the mass density, Young's modulus, and the Poisson ratio for the rubber membrane are $980 \mathrm{~kg} / \mathrm{m}^{3}, 2 \times 10^{5} \mathrm{~Pa}$, and 0.49 , respectively. While Young's modulus and Poisson's ratio for the disk are $2 \times 10^{11} \mathrm{~Pa}$ and 0.29 , respectively. Standard values for air, i.e., $\rho=1.29 \mathrm{~kg} / \mathrm{m}^{3}$, ambient pressure of $1 \mathrm{~atm}$, and speed of sound in air of $c=$ $340 \mathrm{~m} / \mathrm{s}$ were used. It can be seen that there are two transmission peaks at 146 and $974 \mathrm{~Hz}$, with a dip at $272 \mathrm{~Hz}$. These features do not depend on the incidence angle of the sound waves, owing to the orders of magnitude difference between the wavelength of sound in air and the sample size. It is seen that the theoretical predictions agree very well with the experiments under normal incidence.
The effective dynamic mass of the system may be obtained by dividing the averaged stress by the averaged acceleration, i.e., $\rho_{\text {eff }}=\left\langle\sigma_{z z}\right\rangle /\left\langle a_{z}\right\rangle$, with \langle\rangle denoting volume average over the whole membrane structure (membrane plus the weight), while $\sigma_{z z}$ and $a_{z}$ are the stress and acceleration normal to the membrane plane at rest, respectively. Figure 2 shows the results of such calculations. Close to the transmission dip frequency, the effective dynamic mass turns from positive to negative. It then jumps to positive at the dip frequency and then approaches the actual value of the system $\left(\sim 0.1 \mathrm{~kg} / \mathrm{m}^{2}\right)$ at high frequencies. Also plotted in Fig. 2 is the in-plane averaged normal displacement (the dotted green curve), which peaks at the two eigenmodes and goes through zero at the frequency where the transmission is at a minimum. As shown below, there is a link between the two phenomena.

Our calculations also show that the first low-frequency transmission peak is due to the eigenmode in which the membrane and the weight vibrate in unison, while the second transmission peak at high frequency is due to the eigenmode in which the membrane vibrates while the central weight remains almost motionless. As a result, the first peak frequency should depend strongly on the mass of the central weight, while the second peak frequency should have a very weak dependence on the central mass. The experimental transmission spectra for different masses show the same feature of twin peak with a dip in between. The first transmission peak and the dip shift significantly to higher frequencies with the reduction of the mass, while the second transmission peak shifts only by a very small amount. The frequencies of these peaks and the dip are listed in Table I, along with the theoretical results in parenthesis. It is seen that good agreement is achieved between theory and experiments. The discrepancy between the

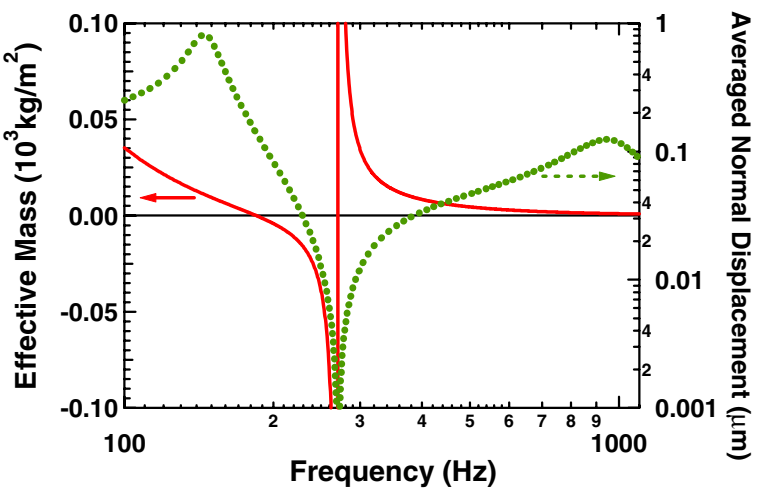

FIG. 2 (color online). The calculated effective dynamic mass of the resonator (red solid curve, left axis) as defined in the text, together with the in-plane averaged normal vibration amplitude (green dotted curve, right axis), evaluated with an incident wave with pressure modulation amplitude of $10^{3} \mathrm{~Pa}$. It is seen that in our system, negative dynamic mass and $\left|\left\langle u_{z}\right\rangle\right| \sim 0$ coincide, and they constitute the basic mechanism for near-total reflection of low-frequency acoustic waves. 
TABLE I. The experimental transmission peak and dip frequencies together with the theoretical values in parentheses.

\begin{tabular}{rccc}
\hline \hline $\begin{array}{c}\text { Mass } \\
(\mathrm{mg})\end{array}$ & $\begin{array}{c}\text { 1st peak frequency } \\
(\mathrm{Hz})\end{array}$ & $\begin{array}{c}\text { Dip frequency } \\
(\mathrm{Hz})\end{array}$ & $\begin{array}{c}\text { 2nd peak frequency } \\
(\mathrm{HZ})\end{array}$ \\
\hline 50 & $286(322)$ & $555(627)$ & $1018(1036)$ \\
100 & $223(242)$ & $376(458)$ & $1000(1000)$ \\
150 & $192(202)$ & $340(378)$ & $976(987)$ \\
200 & $166(177)$ & $284(329)$ & $1025(981)$ \\
250 & $154(159)$ & $259(296)$ & $984(977)$ \\
300 & $145(146)$ & $237(272)$ & $984(974)$ \\
\hline \hline
\end{tabular}

predicted and experimental peak frequencies is less than $10 \%$, while that between the dips is about $20 \%$. The overall trend of the observed variations with the mass of the central weight is very well reproduced by theory.

The frequency trend of the transmission dip is observed to follow that of the first eigenmode. This is understandable, since the vibration amplitude at the dip frequency may be expressed as the superposition of the amplitudes for the two eigenmodes, with an opposite phase [since the dip frequency is higher than the first eigenfrequency but lower than the second, and the resonance response is proportional to $\left(\omega_{i}^{2}-\omega^{2}\right)^{-1}$, with $\omega_{i}$ being the $i$ th eigenfrequency]. The dynamic mass behavior at the dip frequency is directly related to the fact that the integral of the membrane surface's normal displacement passes through zero at that frequency, as seen in Fig. 2. This is easy to see since $\rho_{\text {eff }}^{-1} \propto\left\langle a_{z}\right\rangle \sim \omega^{2}\left\langle u_{z}\right\rangle$, where $u_{z}$ denotes the normal component of the displacement. By decomposing the displacement vector $\vec{u}=\Delta \vec{u}+\left\langle u_{z}\right\rangle$, we may attribute the far-field transmission to $\left\langle u_{z}\right\rangle$ and the near-field effects to $\Delta \vec{u}$. This is because the $\left\langle u_{z}\right\rangle$ part would generate a wave field that has lateral wave vector components peaked at $k_{\|}=0$, and thus the transmitted field due to this "pistonlike" motion will be just a plane wave with the same wave vector as that of the incident wave (since $k_{\|}^{2}+$ $k_{\perp}^{2}=\omega^{2} / c^{2}$, where $c$ is the sound speed in air). In particular, $\left\langle u_{z}\right\rangle=0$ implies total reflection. In contrast, $\Delta \vec{u}$ would generate a wave field that has lateral wave vector components that are peaked at $k_{\|}>\omega / c$ as described below, and hence can only be related to evanescent waves in the transmission direction. In fact, far-field transmittance amplitude may be expressed as

$$
T=\left[1-i\left(2 \rho c \omega \sum_{\alpha} \frac{\left\langle u_{z}^{\alpha}\right\rangle^{2}}{m_{\alpha}\left(\omega_{\alpha}^{2}+2 i b \omega-\omega^{2}\right)}\right)^{-1}\right]^{-1}
$$

where $T$ is the transmission amplitude, $\rho$ the density of air, $b$ the damping coefficient, $\omega$ the frequency of incident wave, and $\omega_{\alpha}, m_{\alpha}$, and $\left\langle u_{z}^{\alpha}\right\rangle$ are the $\alpha$ th eigenfrequency, the generalized mass $\left(m_{\alpha}=\int \rho(\vec{r})\left|\vec{u}_{\alpha}(\vec{r})\right|^{2} d \vec{r}\right.$, with the integral performed over the whole membrane), and the surface integral of the $\alpha$ th eigenmode's normal displacement, respectively.

The near-total reflection by our membrane is not the same as that for a rigid surface, where a node must exist. Because of the weak elastic modulus of the rubber membrane, the tangential wave vector $k_{\|}$of the vibrational eigenmodes is on the same order as the inverse of the membrane radius $r$. Since $r$ is on the order of centimeter, it follows that the wave field generated by the vibrational eigenmodes must have $k_{\|} \gg k_{0}=\omega / c$ in the frequency range of several hundred hertz. As a result, the normal wave vector $k_{\perp}$ is imaginary and $\left|k_{\perp}\right| \gg k_{0}$, which means that in the absence of a nonzero $\left\langle u_{z}\right\rangle$, the acoustic waves should display an evanescent decay over a distance on the order of $2 \pi /\left|k_{\perp}\right|$.

Figure 3 shows the calculated normal velocity field at the dip frequency of $272 \mathrm{~Hz}$ seen in Fig. 1(b). Indeed, the evanescent wave characteristics are seen in the acoustic field distribution near the surfaces of the rubber membrane. In accordance with the simple analysis, we can estimate the decay distance $d$ as

$$
d=\log 2 / \sqrt{\left(\frac{2 \pi}{r}\right)^{2}-\left(\frac{\omega}{c}\right)^{2}} .
$$

For our experimental case with $r=1 \mathrm{~cm}$ and $\omega=$ $272 \mathrm{~Hz}$, the damping distance is about $1.6 \mathrm{~mm}$. This is indeed the case as seen in Fig. 3.

Our membrane-type metamaterial is essentially a twodimensional version of the locally resonant sonic material presented earlier [1]. To compare these two cases, we have performed simulations on the case where a spherical weight is surrounded by rubber [7] in a three-dimensional configuration. Results show that the lowest-frequency eigenmode is one in which the spherical weight and rubber

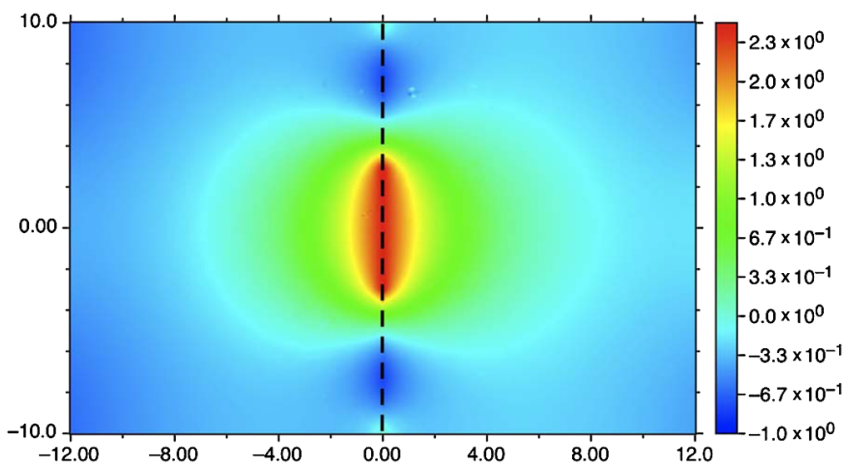

FIG. 3 (color online). The normal velocity field distribution near the membrane at the dip frequency of $272 \mathrm{~Hz}$, where the black dashed line denotes the position of membrane plane. The axes are in units of millimeters, while the velocity is in $\mathrm{mm} / \mathrm{s}$ (calculated with the same incident wave intensity as that for Fig. 2). Damping characteristics near the membrane surfaces indicate a decay length of $1.6 \mathrm{~mm}$, which agrees well with that predicted by Eq. (2). 
vibrate in unison, while the second eigenmode is due almost entirely to rubber vibration only. Between the two eigenmodes there is a transmission dip at which the vibration mode is the mixture of the two. Thus both the 3D and $2 \mathrm{D}$ versions of our metamaterials have the same underlying physics. This understanding is important as it enables the generalization to other material configurations which may also exhibit the negative dynamic mass phenomenon.

Another point to note is that since the negative mass dip in transmission always occurs between the first two eigenmodes, the required elasticity contrast between the central plate (the weight) and the membrane is not very stringent. As long as the frequencies of the bending modes of the central plate, where part of the plate is vibrating out of phase from the other, are well above the first two eigenmodes, the presence of the negative mass dip will not be affected. The bending of the plate could change the frequencies of the two eigenmodes while the plate is vibrating in phase as a whole, which could change the dip frequency but not its existence.

In summary, we have fabricated and characterized a membrane-type metamaterial which demonstrates negative dynamic mass and near-total reflect acoustic waves with frequencies as low as $200-300 \mathrm{~Hz}$. Theoretical understanding of this robust effect is shown to lead to predictions in excellent agreement with the experiments.

Financial support for this work by HKUST 605405 from the Research Grant Council of the Hong Kong SAR gov- ernment is gratefully acknowledged.

*phyang@ust.hk

†sheng@ust.hk

[1] Z. Liu, X. Zhang, Y. Mao, Y. Y. Zhu, Z. Yang, C. T. Chan, and P. Sheng, Science 289, 1734 (2000); Jun Mei, Zhengyou Liu, Weijia Wen, and Ping Sheng, Phys. Rev. B 76, 134205 (2007). The mass density law states that the amplitude transmittance $T$ for the solid panel in air is proportional to the inverse of the product $\rho \ell \omega$, where $\rho$ and $\ell$ are the density and thickness of the solid panel, respectively. Thus low-frequency sound can be very penetrating.

[2] Zhengyou Liu, C. T. Chan, and Ping Sheng, Phys. Rev. B 71, 014103 (2005).

[3] Kin Ming Ho, Chun Kwong Cheng, Z. Yang, X. X. Zhang, and P. Sheng, Appl. Phys. Lett. 83, 5566 (2003).

[4] Zhengyou Liu, C. T. Chan, and Ping Sheng, Phys. Rev. B 62, 2446 (2000).

[5] Jun Mei, Zhengyou Liu, Weijia Wen, and Ping Sheng, Phys. Rev. Lett. 96, 024301 (2006).

[6] Graeme Milton and John Willis, Proc. R. Soc. A 463, 855 (2007).

[7] Kin Ming Ho, Z. Yang, X.X. Zhang, and Ping Sheng, Appl. Acoust. 66, 751 (2005).

[8] See, for example, L. M. Brekhovskikh, Waves in Layered Media (Academic, New York, 1980) 2nd ed. 